\title{
On the Calculation of Average Lifetimes for the 3-body Problem
}

\author{
David Urminsky \\ School of Mathematics and Maxwell Institute for Mathematical Sciences, \\ University of Edinburgh, UK \\ email: david.urminsky@ed.ac.uk
}

\begin{abstract}
Numerical solutions for the 3-body problem can be extremely sensitive to small errors. We consider how small errors in calculations can affect the lifetime of these systems. In particular, we show that numerical errors can shorten the average lifetime of a 3-body system. This is illustrated using the Sitnikov Problem as an example. To give a theoretical explanation, we construct an approximate Poincaré map for this problem and delineate the structure of the escape regions. We show that numerical errors can destroy escape regions and can cause orbits to migrate to a region in which escape is faster.
\end{abstract}

Keywords. stellar dynamics, time

The Sitnikov problem is the problem of the motion of a massless particle, $m_{3}$, on the axis of symmetry of an equal-mass binary (see Moser (1973)). The equation of motion for $m_{3}$ is given by

$$
\ddot{z}=-z /{\sqrt{z^{2}+r^{2}}}^{3},
$$

where $z$ is its position and $r$ the distance from the centre of mass to one of the binary masses. We approximate $r$ to first order in the eccentricity, $e$, by $r \approx \frac{1}{2}(1-e \cos t)$. Taking the plane of motion of the binary $(z=0)$ as a Surface Of Section (SOS), consider a map, $\phi:\left(v_{0}, t_{0}\right) \rightarrow\left(v_{1}, t_{1}\right)$, which takes $m_{3}$ from one crossing of the SOS to the next. If $m_{3}$ is on the SOS at time $t_{0}, \phi$ is a map which brings $v_{0}=\dot{z}\left(t_{0}\right)$ to time $t_{1}>t_{0}$ where $v_{1}=\dot{z}\left(t_{1}\right)$ and $z\left(t_{1}\right)=0$. The map $\phi$ has an open domain $D$ in which every point returns to the SOS. As time enters into the problem with period $2 \pi$, we can consider $D$ in polar co-ordinates where the radial variable is $v$ and the angular variable is given by $t$.

We integrate initial conditions in $D$ forward in time until they satisfy an escape theorem outlined in Urminsky (2007). During the integration we save the time and velocity values for the last 5 crossings of the SOS. Fig. 1(a) shows the structure of these crossings. The crossings form distinct bands which $m_{3}$ visits in turn until finally visiting the upper crescent shaped region before leaving the SOS for the last time. The bands wrap around each other in a fractal like structure spiralling outwards towards the boundary of $D$.

Consider a radial segment, $R$, of initial conditions which traverses these bands and approaches the boundary of $D$. We integrate the initial conditions $R$ forward in time using the Bulirsch-Stoer method for various relative tolerances. Fig. 1(c) shows the average lifetime over $R$ for increasing relative tolerances. There is a clear decrease in the average lifetime as the relative tolerance increases. The plateau for small relative tolerances is due to the maximum time we integrate over and can be increased by integrating longer.

To study the structure of the bands in the SOS we turn to a symplectic approximate Poincaré map (Urminsky (2007)),

$$
\Phi\left(t_{n}, E_{n}\right)=\left\{\begin{array}{l}
t_{n+1}=t_{n}+2 \alpha\left(-E_{n}-a \cos t_{n}-b \sin t_{n}\right)^{-\frac{3}{2}} \\
E_{n+1}=E_{n}+a \cos t_{n}+b \sin t_{n}-a \cos t_{n+1}+b \sin t_{n+1}
\end{array},\right.
$$




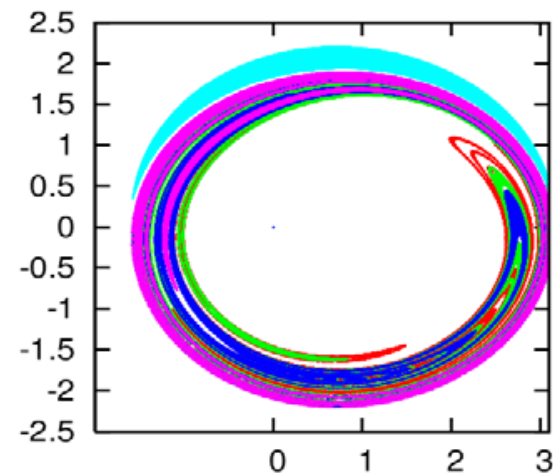

(a)

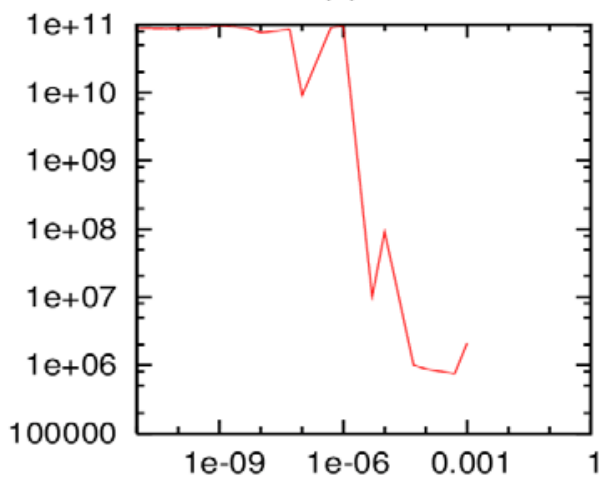

(c)

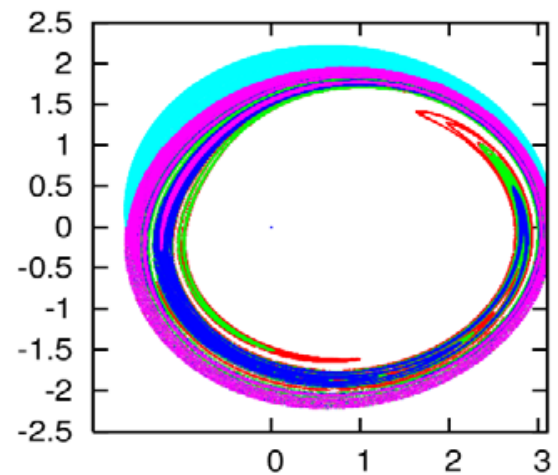

(b)

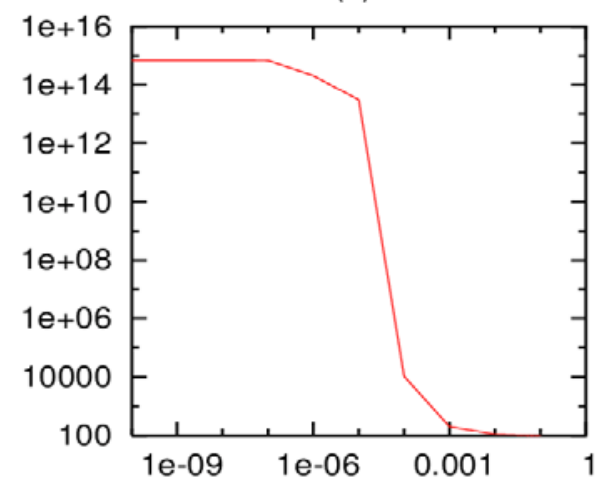

(d)

Figure 1. (a) The last five crossings $m_{3}$ makes with the SOS using equation (0.1) for $e=0.61$. (b) The last five crossings $m_{3}$ makes with the SOS using (0.2) for $e=0.61$. (c) Average lifetime vs. relative tolerance of the Bulirsch-Stoer method using equation (0.1). The maximum time of integration was $10^{11}$. (d) Average lifetime vs. the magnitude of the noise using (0.2).

on an open domain $D^{\dagger}$ where $t_{i}$ represents time at the $i$ th crossing and $E_{i}$ represents the energy of $m_{3}$. The constants $a$ and $b$ are approximately proportional to $e$ and $\alpha=2^{-3 / 2} \pi$. We repeat the experiment in Fig. 1(a) using $\Phi$; the results are displayed in Fig. 1(b). We introduce uniformly distributed noise into (0.2) to mimic errors which would be present by numerically solving (0.1). Varying the magnitude of the noise over a radial segment of initial conditions we find a similar relationship between the average lifetime of orbits and the magnitude of the noise (Fig. 1(d)).

To explain this phenomenon we use $\Phi$ to determine the width of the bands as they wrap around the interior of $D^{\dagger}$. When the width of the bands are comparable to the amplitude of the noise, bands which represent previous crossing blend with successive crossings of the the SOS. This process causes orbits to migrate to bands which lead to quicker escape.

\section{References}

Moser, J. 1973, Stable and Random Motions in Dynamical Systems, Princeton University Press. Urminsky, D. J. 2007, PhD thesis, University of Edinburgh. (in preparation) 